\title{
Pacific
}

Journal of

Mathematics

VALUE DISTRIBUTION OF THE GAUSS MAP AND THE TOTAL CURVATURE OF COMPLETE MINIMAL SURFACE IN $R^{m}$

XIAOKANG Mo

Volume 163 No. 1

March 1994 


\section{VALUE DISTRIBUTION OF THE GAUSS MAP AND THE TOTAL CURVATURE OF COMPLETE MINIMAL SURFACE IN $R^{m}$}

\section{XIAOKANG Mo}

The aim of this paper is to prove the following

THEOREM. Let $S$ be a complete non-degenerate minimal surface in $R^{m}$ such that its generalized Gauss map $f$ intersects only a finite number of times the hyperplanes $A_{1}, \ldots, A_{q}$ in $C P^{m-1}$ in general position. If $q>m(m+1) / 2$, then $S$ must have finite total curvature.

1. Introduction. The study of the value distribution property of Gauss map of minimal surface began with a series of papers by Osserman [9], [11] and the results can be summarized in the following

Theorem ( $R$. Osserman). Let $S$ be a complete minimal surface in $R^{3}$. Then

$S$ has infinite total curvature $\Leftrightarrow$ the Gauss map of $S$ takes on all directions infinitely often with the exception of at most a set of logarithmic capacity zero;

$S$ has finite non-zero total curvature $\Leftrightarrow$ the Gauss map of $S$ takes on all directions a finite number of times, omitting at most three directions;

$S$ has zero total curvature $\Leftrightarrow S$ is a plane.

For a long time, the above theorem had been the best result on this direction. But all the known examples indicated that the exceptional set of logarithmic capacity should be a finite set. In 1981, Xavier made a surprising breakthrough by proving the following result, using a result of Yau about a differential equation on complete Riemannian manifold.

THEOREM (F. Xavier [13]). Let $S$ be a complete minimal surface in $R^{3}$. Then its Gauss map can omit at most six directions unless it is a plane.

In 1988, Fujimoto finally found a way to arrive at the best possible number 4. 
THEOREM (H. Fujimoto [4]). Let $S$ be a complete minimal surface in $R^{3}$. Then its Gauss map can omit at most 4 directions unless it is a plane.

A combination of Osserman's early study and Fujimoto's above work gives the following

Theorem ( $X$. Mo and $R$. Osserman [8]). Let $S$ be a complete minimal surface in $R^{3}$ with infinite total curvature. Then its Gauss map must take every direction infinitely often except at most 4 directions.

For a surface in $R^{m}$ there is the following

THEOREM (H. Fujimoto [5]). Let $S$ be a complete minimal surface in $R^{m}$ with nondegenerate Gauss map. Then the image of $S$ under the Gauss map cannot fail to intersect more than $m(m+1) / 2$ hyperplanes in general position in $C P^{m-1}$.

And the result of this paper mentioned at the beginning of this section is the infinite covering property corresponding to the above theorem.

An oriented minimal surface $S$ in $R^{m}$ may be described by a conformal immersion

$$
X: M \rightarrow R^{m}, \quad X=\left(x_{1}, \ldots, x_{m}\right),
$$

where $M$ is a Riemann surface and each $x_{k}$ is a harmonic function on $M$.

By definition, the generalized Gauss map of $S$ is the map that assigns to each point of $S$ the tangent plane of $S$ at that point. Because the tangent space of $R^{m}$ at every point is naturally identified with $R^{m}$ itself, the range of the Gauss map is the Grassmannian manifold consisting of all the oriented 2-subspaces of $R^{m}$. We can further identify the 2-plane spanned by the orthonormal basis $X, Y$ with the line in $C^{m}$ generated by $(X-i Y) / 2$. So the range of the Gauss map can be thought of as $P^{m-1}(C)$.

Let $z=u+i v$ be a holomorphic local coordinate of $M$. Denote

$$
\frac{\partial x}{\partial z}=\frac{1}{2}\left(\frac{\partial x_{1}}{\partial u}-i \frac{\partial x_{1}}{\partial v}, \ldots, \frac{\partial x_{m}}{\partial u}-i \frac{\partial x_{m}}{\partial v}\right),
$$

by $F=\left(f_{0}, \ldots, f_{n}\right)$, where $n=m-1 ; f=\left(f_{0}: f_{1}: \cdots: f_{n}\right)$ is the point in $C P^{n}$ represented by $\left(f_{0}, \ldots, f_{n}\right)$ in $C^{m}$. Then the 
holomorphic map $f$ represents the Gauss map, and the metric on $M$ as a minimal surface is

$$
d s^{2}=2|F(z)|^{2}|d z|^{2},
$$

where $|F|^{2}=\left|f_{0}\right|^{2}+\cdots+\left|f_{n}\right|^{2}$.

In this way, we turn the problem of the Gauss map partly into a problem on holomorphic curves. The value distribution property of the holomorphic curve may lead to corresponding results about the Gauss map.

In $\S 2$ we will summarize some of the basic ideas and notation of holomorphic curves. We will also introduce an important construction of Cowen and Griffiths [2] on holomorphic curves in $C P^{n}$ which was the basis of their remarkable proof of Ahlfors' defect relation. In $\S 3$ we will present the proof of Fujimoto's inequality in such a way that will clarify the relation between Cowen and Griffiths' construction and Fujimoto's. Fujimoto's inequality is the key to both the proof of his theorem mentioned above and the proof of our result. In $\S 4$, we will give the proof of our result.

2. Some properties of holomorphic curves. Value distribution properties of holomorphic curves have been studied since the end of the 19 th century. The central problem was to generalize the Picard theorem and the Nevanlinna defect relation for entire functions to the case of holomorphic curves. This was finally achieved in 1941 by $\mathrm{L}$. Ahlfors, overcoming great technical difficulties.

In 1976, M. Cowen and P. Griffiths [2] gave a much simpler proof of Ahlfors' result using what they called a "negatively curved collection of metrics". Using their result, H. Fujimoto [5] was able to construct a single metric of negative curvature under certain conditions. Then by the Schwarz-Pick lemma, he derived an inequality which is the key to the study of the value distribution property of the Gauss map of minimal surface. In this section, we will give an outline of Cowen and Griffiths' result.

Let $\Delta_{R}=\{z|| z \mid<R\}$ be a disk in the complex plane, $f: \Delta_{R} \rightarrow$ $P^{n}(C)$ be a holomorphic curve derived from a holomorphic map $F: \Delta_{R} \rightarrow C^{n+1}$ through homogeneous coordinates. $F(z)=\left(f_{0}(z), \ldots\right.$, $\left.f_{n}(z)\right), f_{0}, \ldots, f_{n}$ are holomorphic functions on $\Delta_{R}$. We write $f=$ $\left(f_{0}: \cdots: f_{n}\right)$ and define $|F|=\left(\sum_{i=1}^{n}\left|f_{i}\right|^{2}\right)^{1 / 2}$; for our purposes, we assume that $|F| \neq 0$.

Take the $l$-th derivative:

$$
F^{(l)}(z)=\left(f_{0}^{(l)}(z), \ldots, f_{n}^{(l)}(z)\right) .
$$


Define $F_{k}=F^{(0)} \wedge F^{(l)} \wedge \cdots \wedge F^{(k)}: \Delta_{R} \rightarrow \wedge^{k+1} C^{k+1} \subset G(n, k)$, where $G(n, k)$ is the Grassmannian manifold. By the Plücker embedding $G(n, k) \subset P^{N}(C), N=\left(\begin{array}{c}n+1 \\ k+1\end{array}\right)-1, F_{k}$ induces a map $f_{k}: \Delta_{R} \rightarrow$ $P^{N}(C)$, called the $k$ th derived curve of $f$.

We can define $\left|F_{k}\right|$ in a natural way. Let $e_{0}, \ldots, e_{n}$ be the standard basis of $C^{n+1}$,

$$
F(z)=F^{(0)} \wedge \cdots \wedge F^{(k)}(z)=\sum_{i_{0}<\cdots i_{k}} F_{i_{0}<\cdots<i_{k}} e_{i_{0}} \wedge \cdots \wedge e_{i_{k}},
$$

and we define

$$
\left|F_{k}(z)\right|=\left(\sum_{i_{0}<\cdots<i_{k}}\left|F_{i_{0} \cdots i_{k}}(z)\right|^{2}\right)^{1 / 2} .
$$

Now the Fubini-Study metrics on $P^{n}$ and $P^{N}$ naturally induce metrics on $\Delta_{R}$ by pulling back:

$$
\begin{aligned}
& \Omega_{0}=d d^{c} \log \left|F_{0}\right|^{1 / 2}=d d^{c} \log |F|^{2}, \\
& \Omega_{k}=d d^{c} \log \left|F_{k}\right|^{2}, \quad k=1, \ldots, n,
\end{aligned}
$$

where $d^{c}=(\sqrt{-1} / 4 \pi)(\bar{\partial}-\partial)$. Because $F_{n}$ is just a holomorphic function, $\Omega_{n}=0$. We also set $\left|F_{-1}\right|=1$ for convenience, so $\Omega_{-1}=$ 0 .

The metrics $\Omega_{k}$ will be used later to construct the negatively curved collection of metrics.

Let $a=\left(a_{0}, \ldots, a_{n}\right) \in C^{n+1},|a|=\left(\sum_{i=1}^{n}\left|a_{i}\right|^{2}\right)^{1 / 2}=1$. Then

$$
a_{0} w_{0}+\cdots+a_{n} w_{n}=0
$$

defines a hyperplane, $A$ in both $C^{n+1}$ and $P_{n}$, and

$$
F(A)=a_{0} f_{0}+\cdots+a_{n} f_{n}
$$

measures the distance from $F(z)$ to $A$; in a similar way

$$
\left|F_{k}(A)\right|^{2}=\sum_{i_{1}<\cdots<i_{k}}\left|\sum_{j \neq i_{1}, \ldots, i_{k}} a_{j} F_{j_{i_{1} \cdots i_{k}}}\right|^{2}
$$

measures how far $F_{k}$ is from $A$. Here $F_{j_{i_{1} \cdots i_{k}}}=\operatorname{sign}(\sigma) F_{j_{0} \cdots j_{k}}, \sigma$ is the permutation

$$
\sigma=\left(\begin{array}{ll}
j & i_{1} \cdots i_{k} \\
j_{0} & j_{1} \cdots j_{k}
\end{array}\right)
$$


In fact $\left|F_{k}(A)\left(z_{0}\right)\right|=0$ means $F\left(z_{0}\right), F^{(1)}\left(z_{0}\right), \ldots, F^{(k)}\left(z_{0}\right)$ all lie in the hyperplane

$$
a_{0} w_{0}+\cdots+a_{n} w_{n}=0 .
$$

The corresponding quantities for the holomorphic curve $f$ in $P^{n}$ are

$$
\phi_{0}(A)=\frac{|F(A)|^{2}}{\left|F_{k}\right|^{2}}, \quad \phi_{k}(A)=\frac{\left|F_{k}(A)\right|^{2}}{\left|F_{k}\right|^{2}},
$$

and if $\phi_{k}(A)\left(z_{0}\right)=0$, the curve $f$ is said to have contact of order $k+1$ with $A$ at $z_{0}$.

Now if the holomorphic curve $f: \Delta_{R} \rightarrow P^{n}$ omits a certain number of hyperplanes $A_{1}, A_{2}, \ldots, A_{q}$, we want to construct a metric or a collection of metrics that is negatively curved.

If $n=1, A_{1}, \ldots, A_{q}$ are points on $P^{1}$, we can just pull back the Poincaré metric of $P^{1}-\left\{A_{1}, \ldots, A_{q}\right\}$. To be more explicit, let us take a local coordinate $\zeta$ of $P^{1}$ around a neighborhood of $A_{1}$ (or any other $\left.A_{i}, i=1, \ldots, q\right)$, with $\zeta=0$ at $A^{1}$. Then the Poincaré metric is asymptotically

$$
\frac{d \zeta \wedge d \bar{\zeta}}{|\zeta|^{2} \log ^{2}\left(1 /|\zeta|^{2}\right)}
$$

around the point $A_{1}$. Cowen and Griffiths [2] found a way to generalize this construction to the case when $n \geq 2$. In that case, it becomes necessary to consider not only $f$ but all of its derived curves $f_{k}$. The quantity $|\zeta|^{2}$ for $A_{1}$ will be replaced by $\phi_{k}\left(A_{1}\right)$ as defined above.

Let $\omega=(\sqrt{-1} / 2 \pi) h(z) d z \wedge d \bar{z}$ be a metric. Then the Ricci form is defined by $\operatorname{Ric} \omega=d d^{c} \log h(z)$, and $\operatorname{Ric} \omega \geq \omega$ is equivalent to the fact that the curvature of $\omega$ is less than -1 .

Let $A_{1}, \ldots, A_{q}$ be hyperplanes in general position in $P^{n}$ and $q \geq$ $n+2$. For $i=0, \ldots, n-1$, following the indication of the Poincaré metric, define

$$
\omega_{i}=c_{i} \prod_{\nu=1}^{q}\left(\frac{\phi_{i+1}\left(A_{\nu}\right)}{\phi_{i}\left(A_{\nu}\right) \log ^{2}\left(\mu / \phi_{i}\left(A_{\nu}\right)\right)}\right)^{1 /(n-1)} \Omega_{i} .
$$

Cowen and Griffiths [2] proved the following

Proposition. Given $\varepsilon>0$, for a suitable choice of constants $c_{i}$, and $\mu$, we have

$$
\sum_{i=0}^{n-1}(n-i) \operatorname{Ric} \omega_{i} \geq(q-(n+1)) \Omega_{0}+\sum_{i=0}^{n-1} \omega_{i}-\varepsilon\left(\sum_{i=0}^{n-1} \Omega_{i}\right) .
$$


Aside from the term with the $\varepsilon$, this inequality illustrates what is meant by saying that the collection of metrics $\left\{\omega_{i}\right\}$ is negatively curved. Based on this, Fujimoto constructed a single metric with negative curvature under some additional assumptions. The next section will give a detailed presentation of Fujimoto's construction.

3. Fujimoto's inequality. This section will be centered around curvature computations. For this purpose, a few lemmas from [2] are collected here for convenience.

We have defined $\Omega_{k}=d d^{c} \log \left|F_{k}\right|^{2}$,

LEMMA 1.

$$
\Omega_{k}=\frac{\sqrt{-1}}{2 \pi} \frac{\left|F_{k-1}\right|^{2}\left|F_{k+1}\right|^{2}}{\left|F_{k}\right|^{4}} d z \wedge d \bar{z}
$$

Lemma 2. Define

$$
h_{k}=\frac{\left|F_{k-1}^{2}\right|\left|F_{k+1}\right|^{2}}{\left|F_{k}\right|^{4}} \text {; }
$$

then

$$
\operatorname{Ric} \Omega_{k}=d d^{c} \log h_{k}=\Omega_{k+1}+\Omega_{k-1}-2 \Omega_{k} .
$$

In the process of computation, we will use these two lemmas whenever necessary without referring to them explicitly.

To help understanding, we give here an outline of the idea of the proof of this section. The motivation is to construct a single metric of negative curvature out of a collection of negatively curved metrics.

Let $\omega_{i}=(\sqrt{-1} / 2 \pi) h_{i}(z) d z \wedge d \bar{z}$, and suppose

$$
\sum \operatorname{Ric} \omega_{i} \geq \sum \omega_{i}
$$

Then

$$
\begin{gathered}
\sum_{i} d d^{c} \log h_{i} \geq \sum h_{i} d z \wedge d \bar{z} \\
d d^{c} \log \left(\prod h_{i}\right) \geq\left(\sum h_{i}\right) d z \wedge d \bar{z} \geq n\left(\prod h_{i}\right)^{1 / n} d z \wedge d \bar{z}, \\
d d^{c} \log \left(\prod h_{i}\right)^{1 / n} \geq\left(\prod h_{i}\right)^{1 / n} d z \wedge d \bar{z}
\end{gathered}
$$

so $\omega=\left(\prod h_{i}\right)^{1 / n} d z \wedge d \bar{z}$ satisfies Ric $\omega \geq \omega$ and $\omega$ is the desired metric. In our situation, there are two other factors that complicate the proof. One is that in the proposition of the last section, the collection of metrics is not strictly negatively curved; the term with $\varepsilon$ will cause some complications. The other factor is that there are many 
computations and cancellations due to the special form of metrics that we have. Let us start with the inequality

$$
\sum_{i=0}^{n-1}(n-i) \operatorname{Ric} \omega_{i} \geq(q-(n+1)) \Omega_{0}+\sum_{i=0}^{n-1} \omega_{i}-\varepsilon\left(\sum_{i=0}^{n-1} \Omega_{i}\right),
$$

where

$$
\omega_{i}=c_{i} \prod_{\nu=1}^{q}\left(\frac{\phi_{i+1}\left(A_{\nu}\right)}{\phi_{i}\left(A_{\nu}\right) \log ^{2}\left(\mu / \phi_{i}\left(A_{\nu}\right)\right)}\right)^{1(n-1)} \Omega_{i} .
$$

We want to compute each term of the inequality explicitly.

Step 1.

$$
\begin{aligned}
\sum_{i=0}^{n-1}(n & -i) \operatorname{Ric} \omega_{i} \\
= & \sum_{i=0}^{n-1}(n-i) d d^{c} \log \prod_{\nu=1}^{q}\left(\frac{\phi_{i+1}\left(A_{\nu}\right)}{\phi_{i}\left(A_{\nu}\right) \log ^{2}\left(\mu / \phi_{i}\left(A_{\nu}\right)\right)}\right)^{1 /(n-1)} \\
& +\sum_{i=0}^{n-1}(n-i) \operatorname{Ric} \Omega_{i} \\
= & \sum_{i=0}^{n-1} d d^{c} \log \prod_{\nu=1}^{q} \frac{\phi_{i+1}\left(A_{\nu}\right)}{\phi_{i}\left(A_{\nu}\right) \log ^{2}\left(\mu / \phi_{i}\left(A_{\nu}\right)\right)} \\
& +\sum_{i=0}^{n-1}\left(\Omega_{i+1}+\Omega_{i-1}-2 \Omega_{i}\right) \\
= & d d^{c} \prod_{\nu=1}^{q} \frac{\phi_{n}\left(A_{\nu}\right)}{\phi_{0}\left(A_{\nu}\right) \prod_{i} \log ^{2}\left(\mu / \phi_{i}\left(A_{\nu}\right)\right)}-(n+1) \Omega_{0}
\end{aligned}
$$

but $\phi_{0}\left(A_{\nu}\right)=1, \phi_{0}\left(A_{\nu}\right)=\left|F\left(A_{\nu}\right)\right|^{2} /|F|^{2}$, so

$$
\begin{aligned}
\sum_{i=0}^{n-1}(n-i) \operatorname{Ric} \omega_{i}= & d d^{c} \log \prod_{\nu=1}^{q}\left(\frac{|F|^{2}}{\left|F\left(A_{\nu}\right)\right|^{2} \prod_{i} \log ^{2}\left(\mu / \phi_{i}\left(A_{\nu}\right)\right)}\right) \\
& -(n+1) d d^{c} \log |F|^{2} \\
= & d d^{c} \log \left(\frac{|F|^{2 q}}{\prod_{\nu=1}^{q}\left(\left|F\left(A_{\nu}\right)\right|^{2} \prod_{i} \log ^{2}\left(\mu / \phi_{i}\left(A_{\nu}\right)\right)\right)}\right) \\
& -d d^{c} \log |F|^{2(n+1)} \\
= & d d^{c} \log \left(\frac{|F|^{2(q-(n+1))}}{\prod_{\nu=1}^{q}\left(\left|F\left(A_{\nu}\right)\right|^{2} \prod_{i} \log ^{2}\left(\mu / \phi_{i}\left(A_{\nu}\right)\right)\right)}\right) .
\end{aligned}
$$


Step 2.

$$
\begin{aligned}
\sum_{i=0}^{n-1} \omega_{i} & =\sum_{i=0}^{n-1} c_{i} \prod_{\nu=1}^{q}\left(\frac{\phi_{i+1}\left(A_{\nu}\right)}{\phi_{i}\left(A_{\nu}\right) \log ^{2}\left(\mu / \phi_{i}\left(A_{\nu}\right)\right)}\right)^{1(n-1)} \Omega_{i} \\
& =\sum_{i=0}^{n-1} c_{i} \prod_{\nu=1}^{q}\left(\frac{\phi_{i+1}\left(A_{\nu}\right)}{\phi_{i}\left(A_{\nu}\right) \log ^{2}\left(\mu / \phi_{i}\left(A_{\nu}\right)\right)} h_{i}^{n-i}\right)^{1 /(n-1)} d z \wedge d^{c} z,
\end{aligned}
$$

where $\Omega_{i}=h_{i} d z \wedge d^{c} z$. Using the inequality

$$
a_{1} x_{1}+\cdots+a_{n} x_{n} \geq\left(a_{1}+\cdots+a_{n}\right)\left(x_{1}^{a_{1}} \cdots x_{n}^{a_{n}}\right)^{1 /\left(a_{1}+\cdots+a_{n}\right)}
$$

with $a_{i}=n-i, \sum_{i=0}^{n-1} a_{i}=n(n+1) / 2$, we have

$$
\begin{aligned}
& \sum_{i=0}^{n-1} \omega_{i} \geq C\left(\prod_{i=1}^{n-1}\left(\prod_{\nu=1}^{q}\left(\frac{\phi_{i+1}\left(A_{\nu}\right)}{\phi_{i}\left(A_{\nu}\right) \log ^{2}\left(\mu / \phi_{i}\left(A_{\nu}\right)\right)}\right) h_{i}^{n-i}\right)^{2 / n(n+1)}\right) d z \wedge d^{c} z \\
& =C\left(\prod_{\nu=1}^{q}\left(\frac{\phi_{n}\left(A_{\nu}\right)}{\phi_{0}\left(A_{\nu}\right) \prod_{i=1}^{n-1} \log ^{2}\left(\mu / \phi_{i}\left(A_{\nu}\right)\right)}\right)\right. \\
& \left.\cdot \prod_{i=0}^{n-1}\left(\frac{\left|F_{i-1}\right|^{2}\left|F_{i+1}\right|^{2}}{\left|F_{i}\right|^{4}}\right)^{n-1}\right)^{2 / n(n+1)} \\
& =C\left(\prod_{\nu=1}^{q}\left(\frac{|F|^{2}}{\left|F\left(A_{\nu}\right)\right|^{2} \prod_{i=1}^{n-1} \log ^{2}\left(\mu / \phi_{i}\left(A_{\nu}\right)\right)}\right)\right. \\
& \left.\cdot \prod_{i=0}^{n-1}\left(\frac{\left|F_{n}\right|^{2}}{\left|F_{0}\right|^{2(n+1)}}\right)\right)^{2 / n(n+1)},
\end{aligned}
$$

but $\left|F_{0}\right|=|F|$, so

$$
\sum_{i=0}^{n-1} \omega_{i} \geq C\left(\frac{|F|^{2(q-(n+1))\left|F_{n}\right|^{2}}}{\prod_{\nu=1}^{q}\left(\left|F\left(A_{\nu}\right)\right|^{2} \prod_{i=1}^{n-1} \log ^{2}\left(\mu / \phi_{i}\left(A_{\nu}\right)\right)\right)}\right)^{2 / n(n+1)} .
$$

Step 3.

$$
\begin{aligned}
\varepsilon\left(\sum_{i=1}^{n-1} \Omega_{i}\right) & =\varepsilon \sum_{i=1}^{n-1} d d^{c} \log \left|F_{k}\right|^{2}=d d^{c} \log \left|F_{o}\right|^{2 \varepsilon} \cdots\left|F_{n-1}\right|^{2 \varepsilon}, \\
(q-(n+1)) \Omega_{0} & =(q-(n+1)) d d^{c} \log \left|F_{0}\right|^{2}=d d^{c} \log |F|^{2(q-(n+1))} .
\end{aligned}
$$


Step 4. Combining the results of Steps 1, 2, 3, we have

$$
\begin{aligned}
d d^{c} & \log \frac{\left|F_{0}\right|^{2 \varepsilon} \cdots\left|F_{n-1}\right|^{2 \varepsilon}}{\prod_{\nu=1}^{q}\left(\left|F\left(A_{\nu}\right)\right|^{2} \prod_{i=1}^{n-1} \log ^{2}\left(\mu / \phi_{i}\left(A_{\nu}\right)\right)\right)} \\
& \geq C\left(\frac{|F|^{2(q-(n+1))\left|F_{n}\right|^{2}}}{\prod_{\nu=1}^{q}\left(\left|F\left(A_{\nu}\right)\right|^{2} \prod_{i=1}^{n-1} \log ^{2}\left(\mu / \phi_{i}\left(A_{\nu}\right)\right)\right)}\right)^{2 / n(n+1)} d z \wedge d^{c} z .
\end{aligned}
$$

Setting $G=\prod_{\nu=1}^{q}\left(\left|F\left(A_{\nu}\right)\right|^{2} \prod_{i=1}^{n-1} \log ^{2}\left(\mu / \phi_{i}\left(A_{\nu}\right)\right)\right)$, we have

$$
\begin{aligned}
& d d^{c} \log \left|F_{0}\right|^{2 \varepsilon} \cdots\left|F_{n-1}\right|^{2 \varepsilon}+d d^{c} \log \left(1 / G^{2}\right) \\
& \quad \geq C\left(\frac{|F|^{2(q-(n+1))}\left|F_{n}\right|^{2}}{G^{2}}\right)^{2 / n(n+1)} d z \wedge d^{c} z .
\end{aligned}
$$

Step 5. Notice that $F_{n}$ is a holomorphic function, so $d d^{c} \log |F|=$ 0 ; also $\log |F|^{2}$ is subharmonic, so $d d^{c} \log |F|^{2}$, the $-4 \varepsilon$ in the exponent is necessary and we will see the reason in the arguments later. With $\eta=\left(|F|^{2(q-(n+1))}\left|F_{n}\right|^{2}\right) / G^{2}$, we have

$$
\varepsilon d d^{c} \log \left|F_{0}\right|^{2} \cdots\left|F_{n-1}\right|^{2}+d d^{c} \log \frac{\eta}{|F|^{4 \varepsilon P_{n+1}}} \geq C \eta^{2 / n(n+1)} d z \wedge d^{c} z .
$$

Step 6. Let $P_{n}=n(n+1) / 2, Q_{n}=\sum_{k=1}^{n} P_{k}$. Then

$$
\begin{aligned}
& P_{n} d d^{c} \log \left|F_{0}\right|^{2} \cdots\left|F_{n-1}\right|^{2} \geq\left(P_{n} \frac{\left|F_{1}\right|^{2}}{\left|F_{0}\right|^{4}}+P_{n-1} \frac{\left|F_{0}\right|^{2}\left|F_{2}\right|^{2}}{\left|F_{1}\right|^{4}}+\cdots+P_{1} \frac{\left|F_{n-2}\right|^{2}\left|F_{n}\right|^{2}}{\left|F_{n-1}\right|^{4}}\right) d z \wedge d^{c} z \\
& \geq Q_{n}\left(\left(\frac{\left|F_{1}\right|^{2}}{\left|F_{0}\right|^{4}}\right)^{P_{n}}\left(\frac{\left|F_{0}\right|^{2}\left|F_{2}\right|^{2}}{\left|F_{1}\right|^{4}}\right)^{P_{n-1}}\right. \\
&\left.\cdots\left(\frac{\left|F_{n-2}\right|^{2}\left|F_{n}\right|^{2}}{\left|F_{n-1}\right|^{4}}\right)^{P_{1}}\right)^{1 / Q_{n}} d z \wedge d^{c} z \\
&=Q_{n}\left(\frac{\left|F_{1}\right|^{2} \cdots\left|F_{n-1}\right|^{2}\left|F_{n}\right|^{2}}{\left|F_{0}\right|^{n^{2}+3 n}}\right)^{1 / Q_{n}} d z \wedge d^{c} z,
\end{aligned}
$$

so

$$
\varepsilon d d^{c} \log \left|F_{0}\right|^{2} \cdots\left|F_{n-1}\right|^{2} \geq \varepsilon \frac{Q_{n}}{P_{n}}\left(\frac{\left|F_{0}\right|^{2} \cdots\left|F_{n}\right|^{2}}{\left|F_{0}\right|^{2 P_{n+1}}}\right)^{1 / Q_{n}} d z \wedge d^{c} z
$$


Step 7. Add up the results of Steps 5 and 6, replace the $\varepsilon$ (which is arbitrary) with $\varepsilon / 2$ and notice that $d d^{c} \log \left|F_{n}\right|^{2}=0$, we have

$$
\begin{aligned}
\varepsilon d d^{c} \log \left|F_{0}\right|^{2} \cdots\left|F_{n}\right|^{2}+d d^{c} \log \frac{\eta}{|F|^{2 \varepsilon P_{n+1}}} \\
\geq\left(C \eta^{1 / P_{n}}+\frac{\varepsilon}{2} \frac{Q_{n}}{P_{n}}\left(\frac{\left|F_{0}\right|^{2} \cdots\left|F_{n}\right|^{2}}{\left|F_{0}\right|^{2 P_{n+1}}}\right)^{1 / Q_{n}}\right) d z \wedge d^{c} z,
\end{aligned}
$$

using $a_{1} x_{1}+a_{2} x_{2} \geq\left(a_{1}+a_{2}\right)\left(x_{1}^{a_{1}} x_{2}^{a_{2}}\right)^{1 /\left(a_{1}+a_{2}\right)}$ with $a_{1}=P_{n}, a_{2}=\varepsilon Q_{n}$, we have

$$
d d^{c} \log \frac{\left|F_{0}\right|^{2 \varepsilon} \cdots\left|F_{n}\right|^{2 \varepsilon}}{|F|^{2 \varepsilon P_{n+1}}} \eta \geq C_{1}\left(\frac{\left|F_{0}\right|^{2 \varepsilon} \cdots\left|F_{n}\right|^{2 \varepsilon} \eta}{|F|^{2 \varepsilon P_{n+1}}}\right)^{1 /\left(P_{n}+\varepsilon Q_{n}\right)} d z \wedge d^{c} z
$$

Set

then

$$
h=\left(\frac{\left|F_{0}\right|^{2 \varepsilon} \cdots\left|F_{n}\right|^{2 \varepsilon} \eta}{|F|^{2 \varepsilon M_{n+1}}}\right)^{1 /\left(P_{n}+\varepsilon Q_{n}\right)}
$$

$$
d d^{c} h \geq C_{2} h d z \wedge d^{c} z,
$$

so $h d z \wedge d^{c}<$ is the desired metric.

Step 8. By the Schwarz-Pick lemma, we have a constant $C_{3}$ such that

$$
h(z) \leq C_{3} \frac{2 R}{R^{2}-|z|^{2}},
$$

where $\frac{2 R}{R^{2}-|z|^{2}} d z \wedge d^{c} z$ is the Poincare metric of the disk $\{z|| z \mid<R\}$. Writing out everything explicitly, we have

$$
\frac{|F|^{q-(n+1)-\varepsilon P_{n+1}\left|F_{0}\right|^{\varepsilon} \cdots\left|F_{n-1}\right|^{\varepsilon}\left|F_{n}\right|^{\varepsilon}}}{\prod_{\nu=1}^{q} \prod_{i=1}^{n} \log \left(\mu / \phi_{i}\left(A_{\nu}\right)\right)} \leq C_{4}\left(\frac{2 R}{R^{2}-|z|^{2}}\right)^{P_{n}+Q_{n} \varepsilon} .
$$

Step 9. We would like to get rid of the log terms. Knowing that

$$
K=\sup _{0 \leq x \leq 1} x^{\varepsilon / 2 q} \log \frac{\mu}{x}<+\infty \text { for } \mu>1,
$$

we have

$$
\frac{1}{\log \left(\mu / \phi_{k}\left(A_{\nu}\right)\right)} \geq \frac{1}{K} \phi_{k}\left(A_{\nu}\right)^{\varepsilon / 2 q}=\frac{1}{K} \frac{\left|F_{k}\left(A_{\nu}\right)\right|^{\varepsilon / q}}{\left|F_{k}\right|^{\varepsilon / q}},
$$

substituting this into the result of Step 8, we have

Proposition (Fujimoto's inequality [5]). Let $\Delta_{R}=\{z|| z \mid<R\}$ be a disk in the complex plane, $f: \Delta_{R} \rightarrow C P^{n}$ be a holomorphic curve 
derived from a holomorphic map $F: \Delta_{R} \rightarrow C^{n+1}$, using the notations introduced in the previous section, we have the following statement. For any $\varepsilon>0$, there is a $C>0$, such that

$$
\frac{|F|^{q-(n+1)-\varepsilon P_{n+1}} \prod_{i=1}^{n}\left(\prod_{\nu=1}^{q}\left|F_{k}\left(A_{\nu}\right)\right|\right)^{\varepsilon / q}\left|F_{n}\right|^{1+\varepsilon}}{\prod_{\nu=1}^{q}\left|F\left(A_{\nu}\right)\right|} \leq C\left(\frac{2 R}{R^{2}-|z|^{2}}\right)^{P_{n}+Q_{n} \varepsilon} .
$$

4. Minimal surfaces in $R^{m}$. We assume that all surfaces are orientable, since analogous theorems for non-orientable surfaces are easily formulated by taking the two sheeted orientable covering surface and applying the theorem to it. Following the notation of the previous section, we will prove the following

THEOREM. Let $S$ be a complete non-degenerate minimal surface in $R^{m}$ such that the Gauss map $f=\left(f_{0}: \cdots: f_{n}\right.$ ) (here $n=m=1$ ) intersects only a finite number of times the hyperplanes $A_{1}, \ldots, A_{q}$ (in $\left.C P^{n}\right)$ in general position. If $q>m(m+1) / 2=(n+1)+n(n+1) / 2$, then $S$ must have finite total curvature.

REMARK. If $S$ is a generalized minimal surface with a finite number of branch points, all the arguments of our proof will not be affected. So the theorem is also true for the somewhat more general class of surfaces. This also applies to the similar theorem for surfaces in $R^{3}$ by Mo and Osserman [8].

It was already observed by Osserman (see R. Osserman, $A$ survey of minimal surfaces, second edition, 1986, p. 73) that his classic results on the value distribution of Gauss map is true for simply connected surfaces with a finite number of branch points. An observation of Ahlfors implies that they are still true if a certain condition on the distribution of the branching points is satisfied. But there exist complete generalized minimal surfaces in $R^{3}$, not lying in a plane, whose Gauss map lies in an arbitrarily small neighborhood on the sphere. So the results are not true for arbitrary generalized minimal surfaces. The method of our proof is similar to the method of [8].

Proof. Step 1. Since $f$ is non-degenerate, none of the $F_{k}\left(A_{\nu}\right)$ vanishes identically, where $\nu=1, \ldots, q, k=0, \ldots, n$. Let $A$ be given by the equations

$$
\begin{gathered}
a_{\nu_{0}} z_{0}+\cdots+a_{\nu_{n}} z_{n}=0, \\
F_{k}=\sum_{i_{0}<\cdots<i_{k}} F_{i_{0} \cdots i_{k}} e_{i_{0}} \wedge \cdots \wedge e_{i_{k}},
\end{gathered}
$$




$$
F_{j_{0} \cdots j_{k}}=\operatorname{sign}\left(\begin{array}{c}
i_{0} \cdots i_{k} \\
j_{0} \cdots j_{k}
\end{array}\right) F_{i_{0} \cdots i_{k}}
$$

then for each pair $(\nu, k)$, there is $i_{1}, \ldots, i_{k}$ such that

$$
\psi_{\nu k}=\sum_{l \neq i_{1}, \ldots, i_{k}} a_{\nu l} F_{l_{l_{1} \cdots i_{k}}}
$$

does not vanish identically. Apparently $\psi_{\nu_{0}}=F\left(A_{\nu}\right), \psi_{\nu n}=F_{n}$. Every $\psi_{\nu k}$ is holomorphic, so they have only isolated zeros.

Step 2. The hypothesis of the theorem implies that outside of a compact set $D$ in $S, f$ does not intersect any of the $A_{1}, \ldots, A_{q}$; therefore $F\left(A_{\nu}\right) \neq 0$. Let

$$
S^{\prime}=\left\{p \in S \backslash D: \psi_{\nu k} \neq 0 \text { for any }(\nu, k)\right\} .
$$

On $S^{\prime}$ we define a new metric

$$
d \tilde{s}^{2}=\left|\frac{\prod_{\nu=1}^{q} F\left(A_{\nu}\right)}{\left|F_{n}\right|^{1+\varepsilon} \prod_{\nu, k}\left|\psi_{\nu k}\right|^{\varepsilon / q}}\right|^{2 p^{*}}|d z|^{2}
$$

where

$$
\begin{aligned}
p^{*}= & \frac{1}{\left(q-(n+1)-P_{n+1} \varepsilon\right)-\left(P_{n}+Q_{n} \varepsilon\right)}, \\
& \frac{q-(n+1)-P_{n}}{P_{n+1}+Q_{n}}>\varepsilon>\frac{q-(n+1)-Q_{n}}{P_{n+1}+Q_{n}+1 / q}
\end{aligned}
$$

the last inequality is equivalent to $\varepsilon p^{*} / q>1$.

Here the definition of $d \tilde{s}^{2}$ would be valid if $S^{\prime}$ has a global coordinate $z$. Take a hyperplane $A$ (out of $A_{1}, \ldots, A_{q}$ ). Then on $S^{\prime}$, $f$ does not intersect $A$, namely

$$
a_{0} \frac{\partial x_{1}}{\partial z}+\cdots+a_{n} \frac{\partial x_{n}}{\partial z} \neq 0
$$

this means that if $\xi=a_{0} x_{1}(z)+\cdots+a_{m} x_{m}(z)$ is a global coordinate on $S^{\prime}$, call it $z$, then $d \tilde{s}^{2}$ is well defined.

Step 3. Since $F\left(A_{\nu}\right), F_{n}$ and $\psi_{\nu k}$ are all holomorphic, the metric $d \tilde{s}^{2}$ is flat, and it can be smoothly extended over $D$. We thus obtain a metric, still call it $d \tilde{s}^{2}$, on

$$
S^{\prime \prime}=S^{\prime} \cup D
$$

that is flat outside the compact set $D$. The key to our proof is showing that $S^{\prime \prime}$ is complete in that metric. 
Step 4. We proceed by contradiction. If $S^{\prime \prime}$ is not complete, then there is a divergent curve $\gamma(t)$ on $S^{\prime \prime}$ with finite length. By removing an initial segment, if necessary, we may assume that there is a positive distance $d$ between the curve $\gamma$ and the compact set $D$. Thus $\gamma:[0,1) \rightarrow S^{\prime}$, and since $\gamma$ is divergent on $S^{\prime \prime}$, with finite length, it follows that from the point of view of $S$, either $\gamma(t)$ tends to a point $z_{0}$ where

$$
\left|F_{n}\right|^{1+\varepsilon} \prod_{\nu, k}\left|\psi_{\nu k}\right|^{\varepsilon / q}=0
$$

or else $\gamma(t)$ tends to the boundary of $S$ as $t \rightarrow 1$. But the former case cannot occur, because if

$$
\left|F_{n}\left(z_{0}\right)\right|^{1+\varepsilon} \prod_{\nu, k}\left|\psi_{\nu k}\left(z_{0}\right)\right|^{\varepsilon / q}=0,
$$

then by the fact that $\varepsilon p^{*} / q>1$ (here $q$ is the number of hyperplanes) we have

$$
|d \tilde{s}| \sim \frac{c}{\left|z-z_{0}\right|^{\delta_{0}}} d z
$$

around $z_{0}$ where $c>0, \delta_{0}>1$. Thus

$$
\int_{0}^{1} d \tilde{s}=\infty
$$

contradicting the finite length of $\gamma$.

Step 5. We conclude that $\gamma(t)$ must tend to the boundary of $S$ when $t \rightarrow 1$. Choose $t_{0}$ such that

$$
\int_{t_{0}}^{1} d \tilde{s}<\frac{d}{3}
$$

that is, the length of $\gamma\left(\left[t_{0}, 1\right)\right)$ is less than $d / 3$. Consider a small disk $\Delta$ with center $\gamma\left(t_{0}\right)$. Since $d \tilde{s}^{2}$ is flat, $\Delta$ is isometric to an ordinary disk in the plane. Let $G$ be an isometry of $|w|<\eta$ onto $\Delta$ with $G(0)=\gamma\left(t_{0}\right)$. Extend $G$, as a local isometry into $S^{\prime}$, to the largest disk possible, say $|w|<R$. (Note that $G$ may be viewed simply as the exponential map to $S^{\prime \prime}$ at $\gamma\left(t_{0}\right)$.) In view of $\int_{t_{0}}^{1} d \tilde{s}<\frac{d}{3}$, and the fact that $\gamma$ is a divergent curve on $S$, we have $R \leq d / 3$. Hence the image under $G$ must be bounded away from $D$ by a distance of at least $2 d / 3$. Thus, the reason that the map $G$ cannot be extended to a larger disk must be that the image goes to the boundary of $S^{\prime \prime}$. Since the zeros of $\left|F_{n}\right|^{1+\varepsilon} \prod_{\nu, k}\left|\psi_{\nu k}\right|^{\varepsilon / q}$ have been shown to be infinitely far away in the metric, the image must actually go to the boundary 
of $S$. More specifically, there must be a point $w_{0}$ with $\left|w_{0}\right|=r$, such that the image under $G$ of the line segment from 0 to $w_{0}$ is a divergent curve $\Gamma$ on $S$. Our goal is to show that $\Gamma$ has finite length in the original metric $d s^{2}$ on $S$, contradicting the completeness of the original surface.

Step 6. We know that

$$
|d w|=|d \tilde{s}|=\left|\frac{\prod_{\nu=1}^{q} F\left(A_{\nu}\right)}{\left|F_{n}\right|^{1+\varepsilon} \prod_{\nu, k}\left|\psi_{\nu k}\right|^{\varepsilon / q}}\right|^{p^{*}}|d z| .
$$

Instead of $z$, we change to the coordinate $w$ for the right-hand side of the above expression. Precisely speaking, we let

$$
\bar{F}(w)=\left(\bar{f}_{0}(w), \ldots, \bar{f}_{n}(w)\right)=\left(f_{0}(z(w)), \ldots, f_{n}(z(w))\right)=F(z(w)),
$$

and let $\bar{\psi}_{\nu k}(w)$ be defined from $\bar{F}(z)$ in the same way the $\psi_{\nu k}$ was defined from $F(z)$. Then a little computation shows that

$$
\begin{aligned}
&\left|\frac{d w}{d z}\right|=\left|\frac{\prod_{\nu=1}^{q} \bar{F}\left(A_{\nu}\right)}{\left|\bar{f}_{n}\left(\frac{d w}{d z}\right)^{P_{N}}\right|^{1+\varepsilon} \prod_{\nu, k} \mid \bar{\psi}_{\nu k}\left(\frac{d w}{d z}\right)^{P_{k} \mid \varepsilon / q}}\right|^{P^{*}} \\
&=\left|\frac{\prod_{\nu=1}^{q} \bar{F}\left(A_{\nu}\right)}{\left|\bar{F}_{n}\right|^{1+\varepsilon} \prod_{\nu, k}\left|\bar{\psi}_{\nu k}\right|^{\varepsilon / q}}\right|^{p^{*}} \frac{1}{\left|\frac{d w}{d z}\right|^{p^{*}\left(P_{n}+\varepsilon Q_{n}\right)}}, \\
&\left|\frac{d w}{d z}\right|^{1+p^{*}\left(P_{n}+\varepsilon Q_{n}\right)}=\left|\frac{\prod_{\nu=1}^{q} \bar{F}\left(A_{\nu}\right)}{\left|\bar{F}_{n}\right|^{1+\varepsilon} \prod_{\nu, k}\left|\bar{\psi}_{\nu k}\right|^{\varepsilon / q}}\right|^{p^{*}}
\end{aligned}
$$

by

we have

$$
p^{*}=\frac{1}{\left(q-(n+1)-P_{n+1} \varepsilon\right)-\left(P_{n}+Q_{n} \varepsilon\right)}
$$

$$
\left|\frac{d w}{d z}\right|=\left|\frac{\prod_{\nu=1}^{q} \bar{F}\left(A_{\nu}\right)}{\left|\bar{F}_{n+1}\right|^{1+\varepsilon} \prod_{\nu, k}\left|\bar{\psi}_{\nu k}\right|^{\varepsilon / q}}\right|^{1 /\left(q-(n+1)-P_{n+1} \varepsilon\right)} .
$$

Step 7. We now denote by $C$ the line segment from 0 to $w_{0}$, and by $\Gamma$, the image of $C$ on $S$. Then for the length $L$ of $\Gamma$, we have

$$
\begin{aligned}
L & =2 \int_{C}|F(z(w))||d z(w)| \\
& =2 \int_{C}|\bar{F}(w)|\left|\frac{d z}{d w}\right||d w| \\
& =2 \int\left|\frac{|\bar{F}|^{q-(n+1)-P_{n+1} \varepsilon}\left|\bar{F}_{n+1}\right|^{1+\varepsilon} \prod_{\nu, k}\left|\bar{\psi}_{\nu k}\right|^{\varepsilon / q}}{\prod_{\nu=1}^{q}\left|\bar{F}\left(A_{\nu}\right)\right|}\right|^{1 /\left(q-(n+1)-\varepsilon P_{n+1}\right)} .
\end{aligned}
$$


By the definition of $\bar{\psi}_{\nu k},\left|\bar{\psi}_{\nu k}\right| \leq\left|\bar{F}_{k}\left(A_{\nu}\right)\right|$, and using the proposition of the previous section, the Fujimoto inequality, we have

$$
L \leq 2 C \int\left(\frac{2 R}{R^{2}-|w|^{2}}\right)^{\left(P_{n}+Q_{n} \varepsilon\right) /\left(q-(n+1)-P_{n+1} \varepsilon\right)}|d w| .
$$

Because $0<\left(P_{n}+Q_{n} \varepsilon\right) /\left(q-(n+1)-Q_{n+1} \varepsilon\right)<1, L$ is finite.

Step 8. To sum up, we have shown that if the surface $S^{\prime \prime}$ were not complete, then we could find a divergent curve on $S$ with finite length in the original metric, so that $S$ would not be complete. We therefore conclude that $S^{\prime \prime}$ is complete. Since the metric on $S^{\prime \prime}$ is flat outside of a compact set, we are in a familiar situation (see [11] p. 3564, or Osserman, A survey..., p. 81). By a theorem of Huber [7], the fact that $S^{\prime \prime}$ has finite total curvature implies that $S^{\prime \prime}$ is finitely connected. We conclude first that $\left|F_{n}\right|^{1+\varepsilon} \prod_{\nu, k}\left|\psi_{\nu k}\right|^{\varepsilon / q}$ can have only a finite number of zeros, and second, that the original surface $S$ is finitely connected. Further, by [10, Theorem 2.1] (or the argument in [11, pp. 354]) each annular end of $S^{\prime \prime}$, hence of $S$, is conformally equivalent to a punctured disk. Thus, the Riemann surface $M$ on which $S$ is based must be conformally equivalent to a compact Riemann surface $\bar{M}$ with a finite number of points removed. In a neighborhood of each of those points the Gauss map $f$ does not intersect $q \geq n(n-1) / 2+1 \geq n+2$ hyperplanes. By a generalized Picard theorem (see [2, p. 136]), the Gauss map $f$ can be extended to a holomorphic map from $\bar{M}$ to $P^{n}(C)$. If the homology class represented by the image of $f: \bar{M} \rightarrow P^{n}(C)$ is $m$ times the fundamental homology class of $P^{n}(C)$, then we have

$$
\iint K d A=-2 \pi m
$$

as the total curvature of $S$. This proves the theorem.

Acknowledgment. This paper is the first part of my Ph.D. thesis at Stanford University written under the guidance of Professor R. Osserman. He helped me most generously with his insight into our topics. His exceptional kindness towards a student has encouraged me to complete my work. 


\section{REFERENCES}

[1] S.-S. Chern and R. Osserman, Complete minimal surfaces in euclidean $n$-space, J. Analyse Math., 19 (1967), 15-34.

[2] M. J. Cowen and P. Griffiths, Holomorphic curves and metrics of negative curvature, J. Analyse Math., 29 (1976), 13-153.

[3] H. Fujimoto, On the Gauss map of a complete minimal surface in $R^{m}$, J. Math. Soc. Japan, 35 (1983), 279-288.

[4] _ On the number of exceptional values of the Gauss map of minimal surfaces, J. Math. Soc. Japan, 40 (1988), 235-247.

[5] _ Modified defect relations for the Gauss map of minimal surfaces II, J. Differential Geom., 31 (1990), 365-385.

[6] D. Hoffman and R. Osserman, The geometry of the generalized Gauss map, Mem. Amer. Math. Soc., No. 236, November 1980.

[7] A. Huber, On subharmonic functions and differential geometry in large, Comment. Math. Helv., 32 (1961), 13-72.

[8] X. Mo and R. Osserman, On the Gauss map and total curvature of complete minimal surfaces and an extension of Fujimoto's theorem, J. Differential Geom., 31 (1990), 343-355.

[9] R. Osserman, Proof of a conjecture of Nirenberg, Comm. Pure Appl. Math., 12 (1959), 229-232.

[10] _ On complete minimal surfaces, Arch. Rational Mech. Anal., 13 (1963), 392-404.

[11] _ Global properties of minimal surfaces in $E^{3}$ and $E^{n}$, Ann. of Math., 80 (1964), 340-364.

[12] _ A Survey of Minimal Surfaces, 2nd edition, Dover, New York, 1986.

[13] F. Xavier, The Gauss map of a complete non-flat minimal surface cannot omit 7 points on the sphere, Ann. of Math., 113 (1981), 211-214.

Received December 3, 1990.

WASHINGTON UNIVERSITY

ST. LouIs, MO 63139 


\section{CONTENTS}

P. Ahern and W. Cohn, Weighted maximal functions and derivatives of invariant Poisson integrals of potentials $\ldots, \ldots, \ldots, \ldots, \ldots, \ldots, \ldots, \ldots, \ldots, \ldots$

V. Ancona, T. Peternell, and J. A. Wiśniewski, Fano bundles and splitting theorems

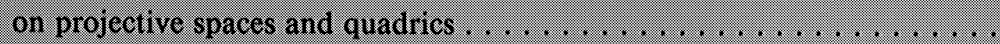

G. M. Beffa, A transverse structure for the Lie-Poisson bracket on the dual of the

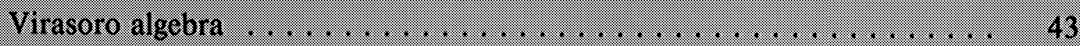

C. Biasi, On ambiental bordism . . . . . . . . . . . . . . . . . 73

P. Debes and M. D. Fried, Nonrigid constructions in Galois theory . . . . . . 81

K. Dykema, Interpolated free group factors . . . . . . . . . . . . . 123

S.-A. Kim and D. Minda, Two-point distortion theorems for univalent functions 137

X. Mo, Value distribution of the Gauss map and the total curvature of complete minimal surface in $R^{m} \ldots \ldots \ldots \ldots \ldots \ldots \ldots \ldots \ldots \ldots$

Z. Shen, On complete Riemannian manifolds with collapsed ends . . . . . . . . 175

F. Zheng, Curvature characterization of certain bounded domains of holomorphy 183

S. Zhu, The classification of complete locally conformally flat manifolds of nonnegative Ricci curvature . . . . . . . . . . . . . . . . . . . . . . 189 


\section{PACIFIC JOURNAL OF MATHEMATICS}

Volume $163 \quad$ No. $1 \quad$ March 1994

Weighted maximal functions and derivatives of invariant Poisson integrals $\quad 1$ of potentials

PATRICK Robert AHERn and WiLliam S. COHN

Fano bundles and splitting theorems on projective spaces and quadrics

VincENZO ANCONA, ThOMAS PETERnELL and JAROSLAW

WISNIEWSKI

A transverse structure for the Lie-Poisson bracket on the dual of the

Virasoro algebra

GLORIA MARI BEFFA

On ambiental bordism

CARLOS BIASI

Nonrigid constructions in Galois theory

PIERRE DÈbes and Michael Fried

Interpolated free group factors

KENNETH JAY DYKEMA

Two-point distortion theorems for univalent functions

SEONG-A KIM and C. DAVID (CARL) MINDA

Value distribution of the Gauss map and the total curvature of complete minimal surface in $R^{m}$

XIAOKANG Mo

On complete Riemannian manifolds with collapsed ends

ZHONGMIN SHEN

Curvature characterization of certain bounded domains of holomorphy

FANGYANG ZHENG

The classification of complete locally conformally flat manifolds of nonnegative Ricci curvature

SHUN-HUi ZHU 